\title{
Estabilidade fenotípica de genótipos de algodoeiro no Estado do Mato Grosso
}

\author{
Eulália Soler Sobreira Hoogerheide(1), Francisco José Correia Farias ${ }^{(2)}$, Roland Vencovsky ${ }^{(1)}$ \\ e Elêusio Curvêlo Freire(2)
}

\begin{abstract}
(1)Escola Superior de Agricultura Luiz de Queiroz, Dep. de Genética e Melhoramento de Plantas, Caixa Postal 83, CEP 13400-970 Piracicaba, SP. E-mail: essobrei@esalq.usp.br, rvencovs@esalq.usp.br (2)Embrapa Algodão, Núcleo do Mato Grosso, Avenida Campo Grande, 612, sala 1, Centro, CEP 78850-000 Primavera do Leste, MT. E-mail: farias@cnpa.embrapa.br, eleusiofreire@hotmail.com
\end{abstract}

Resumo - Foram avaliados oito genótipos de algodoeiro herbáceo, sendo três linhagens e cinco cultivares, com o objetivo de estimar a adaptabilidade e estabilidade fenotípica para o caráter produtividade de algodão em caroço, pelo método Eberhart e Russell. Foram conduzidos 12 experimentos em 11 locais no Estado do Mato Grosso, sob um delineamento de blocos ao acaso com oito repetições, no ano agrícola 2000/2001. Praticamente todos os genótipos apresentaram coeficiente de determinação acima de 85\%, exceto Delta Opal. As estimativas de adaptabilidade indicam que todos os genótipos apresentaram adaptação ampla $\left(b_{i}=1\right)$. Quanto à estabilidade, os genótipos CNPA ITA 90, BRS Antares, CNPA 96-124, CNPA 96-283 e BRS Aroeira revelaram-se estáveis $\left(\mathrm{s}_{\mathrm{d}_{\mathrm{i}}}^{2}=0\right)$. Os melhores genótipos, caracterizados pela maior produtividade, estabilidade e adaptabilidade ampla foram CNPA ITA 90, BRS Aroeira e CNPA 96-124.

Termos para indexação: adaptabilidade, produtividade, interação genótipo x ambiente.

\section{Phenotypic stability in cotton genotypes in Mato Grosso State, Brazil}

\begin{abstract}
Eight cotton genotypes, three lines and five cultivars, were evaluated for estimation of phenotypic adaptability and stability parameters relative to cotton yield using the method proposed by Eberhart and Russell. Twelve yield trials, in randomized complete blocks, comprising eight replications, were carried out in 11 locations of the Mato Grosso State, during the 2000/2001 crop season. All the genotypes showed determination coefficient above of $85 \%$, except Delta Opal. For the estimates of adaptability, all the genotypes presented broad adaptation $\left(b_{i}=1\right)$. The genotypes CNPA ITA 90, BRS Antares, CNPA 96-124, CNPA 96-283 and BRS Aroeira showing hight stability $\left(\mathrm{s}_{\mathrm{d}_{\mathrm{i}}}^{2}=0\right)$. The best genotypes, characterized by higher yield, stability and broad adaptability, were CNPA ITA 90, BRS Aroeira and CNPA 96-124.
\end{abstract}

Index terms: adaptability, yield, genotype x environment interaction.

\section{Introdução}

O Estado do Mato Grosso possui excelente condição climática para cultivo do algodão. As propriedades agrícolas se caracterizam por cultivar grandes áreas, 100 a 5.000 ha, usar a mecanização do plantio à colheita, e utilizar intensivamente insumos, tais como corretivos, adubos químicos, herbicidas, fungicidas, reguladores de crescimento e desfolhantes. Devido às condições favoráveis e à extensa área cultivada, o Mato Grosso, além de maior produtor nacional, é detentor da maior produtividade média nacional, com $3.600 \mathrm{~kg} \mathrm{ha}^{-1}$ de algodão em caroço (Conab, 2006).

Apesar da elevada produtividade, o custo de produção da cultura é considerado alto, o que pode inviabilizar o seu cultivo. Uma das alternativas que vêm sendo recomendada, visando à diminuição contínua do custo de produção, é o emprego de cultivares produtivas e resistentes às principais doenças de ocorrência na região (Farias, 2005).

Devido às extensas áreas cultivadas, à diversidade das condições edafoclimáticas e às variações das técnicas de manejo, espera-se elevada interação genótipo $\mathrm{x}$ ambiente (GxA), o que dificulta a identificação e recomendação de materiais produtivos e estáveis (Farias et al., 1996). Campbell \& Jones (2005) definem interação GxA como a resposta diferencial dos genótipos para um dado caráter em diferentes ambientes.

A interação GxA é um importante componente de programas de melhoramento de plantas para o 
desenvolvimento de cultivares (Campbell \& Jones, 2005). Para avaliar essa interação, a condução de experimentos num maior número de locais possíveis é fundamental, para se quantificar os efeitos da interação nas características agronômicas desejáveis da planta e posterior recomendação dos genótipos para cultivo (Farias et al., 1996).

$\mathrm{O}$ estudo da estabilidade e adaptabilidade é uma maneira de avaliar o fenômeno da interação entre genótipos e ambientes sendo de importância capital para o melhorista, cujo interesse maior é a obtenção de materiais que se comportem bem não somente em um ambiente particular, mas também sob diferentes condições ambientais. Segundo Cruz \& Regazzi (2001), entende-se por adaptabilidade a capacidade de os materiais aproveitarem vantajosamente o estímulo ambiental, ao passo que a estabilidade indica a capacidade dos mesmos mostrarem um comportamento altamente previsível de acordo com o ambiente.

O estudo da estabilidade e adaptabilidade fenotípica tem sido uma estratégia bastante utilizada em várias espécies, com destaque para o arroz (Santos et al., 2005), milho (Carvalho et al., 2005), alfafa (Ferreira et al., 2004), feijão (Carbonell et al., 2001) e girassol (Lúquez et al., 2002). Quanto à cultura do algodão, diversos resultados foram obtidos (Silva et al., 1995; Scapim et al., 2000; Hoogerheide, 2004; Campbell \& Jones, 2005; Farias, 2005).

Entre os métodos utilizados, destaca-se o de Eberhart \& Russell (1966), um dos mais utilizados, o qual baseiase na análise de um coeficiente de regressão linear e de uma variância dos desvios da regressão, que são estimados para cada genótipo.

O trabalho teve como objetivo avaliar a estabilidade e adaptabilidade fenotípica para o caráter produtividade em caroço de genótipos de algodão em diferentes locais do Estado do Mato Grosso.

\section{Material e Métodos}

Oito genótipos de algodoeiro foram avaliados: cinco cultivares (CNPA ITA 90, BRS Antares, Delta Opal, BRS 197 e BRS Aroeira) e três linhagens (CNPA 96-227, CNPA 96-124, CNPA 96-283), em 12 experimentos do ensaio final de genótipos de algodoeiro conduzido pela Embrapa Algodão no Estado do Mato Grosso, no ano agrícola 2000/2001. Os experimentos foram conduzidos em 11 locais, e no Município de Campo Novo do Parecis foram avaliados dois experimentos. As coordenadas geográficas e altitude dos locais encontram-se na Tabela 1.
O delineamento experimental utilizado foi o de blocos ao acaso com oito repetições. A parcela experimental foi constituída de quatro fileiras de $5 \mathrm{~m}$, com espaçamento de 0,90 a $1,0 \mathrm{~m}$ entre fileiras e sete plantas por metro. A área útil da parcela foi composta por duas fileiras centrais. Na avaliação da produtividade foi colhido o algodão em caroço na área útil.

Realizaram-se as análises individuais por local e, em seguida, procedeu-se à análise de variância conjunta, considerando fixo o efeito de genótipos e aleatório o de locais. Após a análise conjunta, realizou-se o estudo de adaptabilidade e estabilidade utilizando o método de Eberhart \& Russell (1966). Este método baseia-se no seguinte modelo de regressão linear:

$\overline{\mathrm{Y}}_{\mathrm{ik}}=\mathrm{m}_{\mathrm{i}}+\mathrm{b}_{\mathrm{i}} \mathrm{I}_{\mathrm{k}}+\mathrm{d}_{\mathrm{ik}}+\overline{\mathrm{e}}_{\mathrm{ik}}$, em que: $\overline{\mathrm{Y}}_{\mathrm{ik}}$ é a média do tratamento i no local $\mathrm{k} ; \mathrm{m}_{\mathrm{i}}$ é a média geral do tratamento $\mathrm{i} ; \mathrm{b}_{\mathrm{i}}$ é o coeficiente de regressão linear, que mede a resposta do genótipo i à variação dos locais; $\mathrm{I}_{\mathrm{k}}$ é o índice ambiental para o local $\mathrm{k}$; $\mathrm{d}_{\mathrm{ik}}$ mede o desvio da regressão do tratamento i no local $\mathrm{k}$; $\overline{\mathrm{e}}_{\mathrm{ik}}$ é o erro experimental médio.

$\mathrm{O}$ parâmetro $\mathrm{s}_{\mathrm{d}_{\mathrm{i}}}^{2}$ mensura a variância dos desvios da regressão e avalia a capacidade da cultivar apresentar comportamento previsível em função do estímulo ambiental. O parâmetro $b_{i}$ é o coeficiente da regressão linear, que indica a adaptabilidade do material. São considerados de adaptabilidade geral os genótipos que apresentarem $b_{i}=1$; adaptabilidade específica a ambientes favoráveis, aqueles com $b_{i}$ maior que 1 ; e adaptabilidade específica a ambientes desfavoráveis, aqueles com $b_{i}$ menor que 1.

A cultivar adaptada e estável, segundo este método, é a que apresenta $b_{i}=1$ e $s_{\mathrm{d}_{\mathrm{i}}}^{2}=0$, respectivamente.

Tabela 1. Coordenadas geográficas e altitude dos municípios onde foram conduzidos os experimentos do Ensaio Final de Algodoeiro Herbáceo no Estado do Mato Grosso, no ano agrícola 2000/2001.

\begin{tabular}{lccc}
\hline Município & Latitude $(\mathrm{S})$ & Longitude $(\mathrm{W})$ & Altitude (m) \\
\hline Alto Taquari & $17^{\circ} 49^{\prime} \mathrm{S}$ & $53^{\circ} 16^{\prime} \mathrm{W}$ & 851 \\
Campo Novo do Parecis ${ }^{(1)}$ & $14^{\circ} 54^{\prime} \mathrm{S}$ & $53^{\circ} 01^{\prime} \mathrm{W}$ & 572 \\
Campo Verde & $15^{\circ} 32^{\prime} \mathrm{S}$ & $55^{\circ} 10^{\prime} \mathrm{W}$ & 736 \\
Rondonópolis & $16^{\circ} 28^{\prime} \mathrm{S}$ & $54^{\circ} 38^{\prime} \mathrm{W}$ & 520 \\
Nova Mutum & $13^{\circ} 49^{\prime} \mathrm{S}$ & $56^{\circ} 04^{\prime} \mathrm{W}$ & 460 \\
Sapezal & $14^{\circ} 54^{\prime} \mathrm{S}$ & $53^{\circ} 01^{\prime} \mathrm{W}$ & 370 \\
Pedra Preta & $16^{\circ} 37^{\prime} \mathrm{S}$ & $54^{\circ} 28^{\prime} \mathrm{W}$ & 850 \\
Primavera do Leste & $15^{\circ} 33^{\prime} \mathrm{S}$ & $54^{\circ} 17^{\prime} \mathrm{W}$ & 465 \\
Itiquira & $17^{\circ} 12^{\prime} \mathrm{S}$ & $54^{\circ} 09^{\prime} \mathrm{W}$ & 522 \\
Lucas do Rio Verde & $13^{\circ} 03^{\prime} \mathrm{S}$ & $55^{\circ} 54^{\prime} \mathrm{W}$ & 390 \\
Sorriso & $12^{\circ} 37^{\prime} \mathrm{S}$ & $57^{\circ} 29^{\prime} \mathrm{W}$ & 365 \\
\hline
\end{tabular}

${ }^{(1)}$ Local onde foram conduzidos dois experimentos. 
A hipótese de que o coeficiente de regressão não difere da unidade e os desvios da regressão não diferem de zero foi avaliada pelo teste te F, respectivamente. Além disso, para atender os objetivos do programa de melhoramento de algodão, espera-se obter genótipos que apresentem elevada produtividade.

\section{Resultados e Discussão}

O coeficiente de variação (CV) ambiental, que é a precisão experimental média, foi de $14,6 \%$, para a análise de variância conjunta da produtividade de algodão em caroço (Tabela 2). Em estudos da mesma natureza, Campbell \& Jones (2005) e Farias (2005) obtiveram CV médio de 15,2 e $12,1 \%$, respectivamente. Isso indicou que os experimentos desta pesquisa apresentaram uma precisão satisfatória.

A fonte de variação da interação genótipos x locais foi altamente significativa $(p<0,01)$, o que indica que os materiais não se comportaram de modo consistente nos diferentes locais, justificando assim o estudo mais detalhado a fim de identificar os genótipos de maior adaptabilidade e estabilidade.

Na Tabela 3 encontram-se os parâmetros estimados pelo método de Eberhart \& Russell (1966). Praticamente

Tabela 2. Análise de variância conjunta para a produtividade em caroço $\left(\mathrm{kg} \mathrm{ha}^{-1}\right)$ dos experimentos do ensaio final de algodoeiro herbáceo no Estado do Mato Grosso, no ano agrícola 2000/2001.

\begin{tabular}{lcc}
\hline Fonte de variação & GL & Quadrado médio \\
\hline Locais (L) & 11 & $61.624 .141,1^{* *}$ \\
Genótipos (G) & 7 & $3.616 .118,2^{* *}$ \\
GxL & 77 & $846.488,8^{* *}$ \\
Resíduo & 588 & $255.535,2$ \\
\hline CV (\%) & 14,6 & \\
Média & 5.223 & \\
**Significativo 1\% de probabilidade, pelo teste F.
\end{tabular}

todos os genótipos apresentaram coeficiente de determinação $\left(\mathrm{R}^{2}\right)$ acima de $85 \%$, exceto Delta Opal. De modo geral, os genótipos apresentaram comportamentos previsíveis ou estáveis diante das variações ambientais, conforme Cruz \& Regazzi (2001). Tais resultados estão de acordo com os obtidos por Farias (2005) e Suinaga et al. (2006). No genótipo Delta Opal, o $\mathrm{R}^{2}$ foi $74 \%$, o que mostrou ajustamento inadequado dos dados à reta de regressão, evidenciando baixa previsibilidade de comportamento nos ambientes avaliados, como foi confirmado pelas estimativas dos desvios da regressão $\left(\mathrm{s}_{\mathrm{d}_{\mathrm{d}}}^{2}\right)$.

As estimativas dos parâmetros de adaptabilidade indicam que todos os genótipos apresentaram adaptação ampla $\left(b_{i}=1\right)$, ou seja, responderam da mesma forma tanto para ambientes desfavoráveis quanto favoráveis. Estudos dessa mesma natureza foram realizados por Hoogerheide (2004), no Estado do Mato Grosso. Este autor observou estimativa de $b=1$ para CNPA ITA 90, coincidindo assim com as alcançadas neste trabalho, confirmando a adaptabilidade ampla desse material. Entretanto, para o genótipo Delta Opal, Hoogerheide (2004) obteve estimativa de b>1, o que demonstra adaptação a ambientes favoráveis.

Quanto à estabilidade, medida pelos desvios da regressão $\left(\mathrm{s}_{\mathrm{d}}^{2}\right)$, os genótipos Delta Opal, BRS 197 e CNPA 96-227 foram considerados instáveis $\left(\mathrm{s}_{\mathrm{d}_{\mathrm{i}}}^{2} \neq 0\right)$. Estas estimativas coincidem com as obtidas por Hoogerheide (2004) para Delta Opal. Os genótipos CNPA ITA 90, BRS Antares, CNPA 96-124, CNPA 96-283 e BRS Aroeira revelaram-se estáveis $\left(\mathrm{s}_{\mathrm{d}_{\mathrm{i}}}^{2}=0\right)$, não diferindo significativamente. Em estudos realizados por Hoogerheide (2004), a cultivar CNPA ITA 90 apresentou baixa previsibilidade nos ambientes, não coincidindo, portanto, com as estimati-

Tabela 3. Estimativas dos parâmetros de estabilidade e adaptabilidade para produtividade em caroço $\left(\mathrm{kg} \mathrm{ha}^{-1}\right)$, pelo método de Eberhart e Russel, dos experimentos do ensaio final de algodoeiro herbáceo no Estado do Mato Grosso, no ano agrícola 2000/ $2001^{(1)}$.

\begin{tabular}{|c|c|c|c|c|}
\hline Genótipo & Média & $b_{i}$ & $\mathrm{R}^{2}(\%)$ & $\mathrm{s}_{\mathrm{d}_{\mathrm{i}}}^{2}$ \\
\hline CNPA ITA 90 & $5.441,00 \mathrm{a}$ & $0,98^{\mathrm{ns}}$ & 98 & $-7.254,3^{\mathrm{ns}}$ \\
\hline BRS Antares & $5.155,00 \mathrm{bc}$ & $1,01^{\mathrm{ns}}$ & 97 & $5.308,7^{\mathrm{ns}}$ \\
\hline Delta Opal & $5.060,05 \mathrm{~cd}$ & $0,93^{\mathrm{ns}}$ & 74 & $294.615,5^{* *}$ \\
\hline BRS 197 & $5.393,10 \mathrm{a}$ & $1,05^{\mathrm{ns}}$ & 87 & $150.073,5^{* *}$ \\
\hline CNPA 96-227 & $4.893,20 \mathrm{~d}$ & $1,09^{\mathrm{ns}}$ & 91 & $92.754,9^{*}$ \\
\hline CNPA 96-124 & $5.302,25 \mathrm{ab}$ & $0,94^{\mathrm{ns}}$ & 96 & $4.282,3^{\mathrm{ns}}$ \\
\hline CNPA 96-283 & $5.172,31 b c$ & $0,92^{\mathrm{ns}}$ & 99 & $-22.521,6^{\mathrm{ns}}$ \\
\hline$\underline{B R S}$ Aroeira & $5.420,41 \mathrm{a}$ & $1,04^{\mathrm{ns}}$ & 96 & $12.982,2^{\mathrm{ns}}$ \\
\hline
\end{tabular}

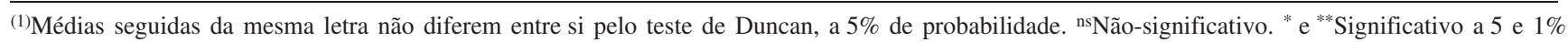
de probabilidade, respectivamente. 
vas deste trabalho. Tais discrepâncias são esperadas, pois, de acordo com o descrito por Crossa (1990), os resultados das estimativas de adaptabilidade e estabilidade são relativos aos ambientes e ao grupo de cultivares envolvidos, não podendo ser generalizados, o que indica que uma cultivar estável num determinado grupo não o será necessariamente em outro.

As cultivares que se destacaram devido à estabilidade $\left(\mathrm{s}_{\mathrm{d}_{\mathrm{i}}}^{2}=0\right)$, ampla adaptação $\left(\mathrm{b}_{\mathrm{i}}=1\right)$ e elevada produtividade foram CNPA ITA 90, BRS Aroeira e CNPA 96-124 (Tabela 3). Assim, percebe-se o desempenho consistente e com alta produtividade desses genótipos nos ambientes. De acordo com Kang (1998), isso permite ampla e durável resistência e tolerância aos fatores bióticos e abióticos sob os quais os genótipos se desenvolveram.

\section{Conclusões}

1. A interação significativa genótipo $x$ ambiente indica que o comportamento dos genótipos não é consistente nos diferentes locais.

2. Os genótipos CNPA ITA 90, BRS Aroeira e CNPA 96-124 são amplamente adaptados, estáveis e produtivos para as condições ambientais do Estado do Mato Grosso.

\section{Agradecimentos}

Ao CNPq, pela concessão da bolsa de estudo ao primeiro autor; à Embrapa Algodão, pelo fornecimento dos dados.

\section{Referências}

CAMPBELL, B.T.; JONES, M.A. Assessment of genotype $\mathrm{x}$ environment interactions for yield and fiber quality in cotton performance trials. Euphytica, v.144, p.69-78, 2005.

CARBONELL, S.A.M.; AZEVEDO FILHO, J.A. de; DIAS, L.A.S.; GONÇALVES, C.; ANTONIO, C.B. Adaptabilidade e estabilidade de produção de cultivares e linhagens de feijoeiro no Estado de São Paulo. Bragantia, v.60, p.69-77, 2001.

CARVALHO, H.W.L. de; CARDOSO, M.J.; LEAL, M.L.S.; SANTOS, M.X. dos; TABOSA, J.N.; SOUZA, E.M. de. Adaptabilidade e estabilidade de cultivares de milho no Nordeste brasileiro. Pesquisa Agropecuária Brasileira, v.40, p.877-883, 2005.
CONAB. Levantamento de outubro de 2006. Disponível em: $<$ http://www.conab.gov.br/conabweb/index.php?PAG=131>. Acesso em: 3 jan. 2007.

CROSSA, J. Statistical analysis of multilocation trials. Advances in Agronomy, v.44, p.55-85, 1990.

CRUZ, C.D.; REGAZZI, A.J. Modelos biométricos aplicados ao melhoramento genético. Viçosa: UFV, 2001. 390p.

EBERHART, S.A.; RUSSEL, W.A. Stability parameters for comparing varieties. Crop Science, v.6, p.36-40, 1966.

FARIAS, F.J.C. Índice de seleção em cultivares de algodoeiro. 2005. 121p. Tese (Doutorado) - Escola Superior de Agricultura Luiz de Queiroz, Piracicaba.

FARIAS, F.J.; FREIRE, E.C.; CARVALHO, L.P. de; ARANTES, E.M.; OLIVEIRA, L.C. de. Estabilidade e adaptabilidade de genótipos de algodoeiro herbáceo no Estado do Mato Grosso. Campina Grande: Embrapa-CNPA, 1996. 4p. (Embrapa-CNPA. Pesquisa em andamento, 29).

FERREIRA, P. de R.; BOTREL, M. de A.; RUGGIERI, A.C.; PEREIRA, A.V.; COELHO, A.D.F.; LÉDO, F.J. da S.; CRUZ, C.D. Adaptabilidade e estabilidade de cultivares de alfafa em relação a diferentes épocas de corte. Ciência Rural, v.34, p.265-269, 2004.

HOOGERHEIDE, E.S.S. Estabilidade fenotípica de algodoeiro herbáceo em diferentes sistemas de produção no Estado do Mato Grosso. 2004. 80p. Dissertação (Mestrado) - Escola Superior de Agricultura Luiz de Queiroz, Piracicaba.

KANG, M.S. Using genotype-by-environment interaction for crop cultivar development. Advances in Agronomy, v.62, p.199-252, 1998.

LÚQUEZ, J.E.; AGUIRREZÁBEL, L.A.N.; AGUERO, M.E.; PEREYRA, V.R. Stability and adaptability of cultivars in nonbalanced yield trials. Comparison of methods for selecting high oleic sunflower hybrids for grain yield and quality. Journal of Agronomy and Crop Science, v.188, p.225-234, 2002.

SANTOS, P.G.; MELO, L.C.; SOARES, A.A.; REIS, M.S.; JULIATTI, F.C.; CORNÉLIO, V.M.O. Study of the interaction genotypes $\mathrm{x}$ environments in the selection process of upland rice. Crop Breeding and Applied Biotechnology, v.5, p.38-46, 2005.

SCAPIM, C.A.; OLIVEIRA, V.R.; BRACCINI, A. de L. e; CRUZ, C.D.; ANDRADE, C.A. de B.; VIDIGAL, M.C.G. Yield stability in maize (Zea mays L.) and correlations among the parameters of the Eberhart and Russell, Lin and Binns and Huehn models. Genetics and Molecular Biology, v.23, p.387-393, 2000.

SILVA, F.G.; ANUNCIAÇÃO FILHO, C.J.; TABOSA, J.N. Estabilidade da produção de grãos de arroz irrigado nos Estados de Alagoas e de Pernambuco. Pesquisa Agropecuária Brasileira, v.30, p.347-351, 1995.

SUINAGA, F.A.; BASTOS, C.S.; RANGEL, L.E.P. Phenotypic adaptability and stability of cotton cultivars in Mato Grosso State, Brazil. Pesquisa Agropecuária Tropical, v.36, p.145-150, 2006.

Recebido em 6 de junho de 2006 e aprovado em 28 de março de 2007 